\title{
Special Issue on 20 Years of Multiple-Point Statistics: Part 2
}

\author{
Grégoire Mariethoz • Philippe Renard
}

Published online: 12 June 2014

(C) International Association for Mathematical Geosciences 2014

While the first part of this special issue on Multiple Point Statistics (Issue 2, February 2014) is devoted to theoretical advances, this second part focuses on applications. It is notable that in its early days MPS was mostly considering the realm of synthetic cases, with many publications focusing on new algorithms of increased complexity and minutiae of implementation descriptions. This phase was necessary to develop the essential concepts and algorithmic tools that can make the MPS idea applicable today. There is now a steep rise in the number of applications of MPS, which occurs in several different domains of geosciences. This special issue reflects this increase, to present case studies ranging from geophysics, water resources and mineral deposit modeling. In addition, it includes a strong emphasis on inverse modeling with training images, which remained a prominent question since the early days of MPS.

The present volume starts with a paper by Marijke Huysmans, Philippe Orban, Elke Cochet, Mathias Possemiers, Benedicta Ronchi, Katherine Lauriks, Okke Batelaan and Alain Dassargues. The paper focuses on the accurate reproduction of the contacts between geological bodies in a hydrogeological context. The method of edge properties simulation is used, which is based on a change of variable that transforms a categorical pixel-based training image into a description of the interfaces between facies that occur at sub-pixel scale. Cross-bedded aquifer sand bodies are modeled and the influence of the edge properties on flow and transport processes is quantified.

G. Mariethoz $(\bowtie)$

School of Civil and Environmental Engineering, The University of New South Wales,

Sydney, Australia

e-mail: gregoire.mariethoz@minds.ch

P. Renard

Centre of Hydrogeology and Geothermics, University of Neuchâtel,

11 Rue Emile Argand, 2000 Neuchâtel, Switzerland

e-mail: philippe.renard@unine.ch 
Next is a second paper focusing on hydrogeology by James L. McCallum, Daan Herckenrath and Craig Simmons. It investigates the reproduction of connectivity patterns by different geostatistical simulation methods, and under various amounts of conditioning data. A particular aspect is that the effect of aquifer connectivity is quantified using groundwater residence time distributions.

The third paper of this issue is by Alexandre Boucher, Joao Felipe Costa, Luis Gustavo Rasera and Eduardo Motta that presents an application in simulating mineral deposits. The modeling approach adopted consists in using an existing deterministic model the studied domain as training image. MPS is then used to re-simulate only the zones of the contacts between facies, since geological uncertainty in the deposit under study relates to those areas.

The three remaining papers of this issue are specifically focusing on the resolution of inverse problems in the context of MPS. One difficulty in this domain is that it is often difficult to use classical inversion methods because MPS deal with non-smooth fields, whose hydraulic behavior can be unstable, with minute spatial perturbations having unpredictable consequences in terms of flow and transport. Morteza Khodabakhshi and Behnam Jafarpour present a method where the history matching procedure efficiency is enhanced by keeping track of its past iterations using a "memory map". An interesting aspect of the approach is that it is able to use several candidate training images during the inversion process.

The last two papers of this special issue propose another approach to inverse problems based on a pattern searching procedure carried out through an ensemble of training images. The methodology is general because the training images can be obtained by using a deterministic physical simulation. It is interesting that a similar methodology has been applied in two different fields. The paper by Liangping Li, Sanjay Srinivasan, Haiyan Zhou and Jaime Gómez-Hernández use flow simulation to relate the spatial features of hydraulic head with patterns of hydraulic conductivity. On the other hand, the paper by Tobias Lochbühler, Guillaume Pirot, Julien Straubhaar and Niklas Linde focuses on geophysical inverse problems, a topic that has not yet been thoroughly investigated in the context of MPS, and compares the distribution of facies with patterns in the GPR wave velocities. In both cases, the ensemble of training images is used to generate an ensemble of realizations that are conditioned to dynamic data.

We hope that the applications presented in this issue will constitute a useful guide for practitioners and modelers who wish to employ training images in various fields of geosciences for quantifying uncertainty in spatial phenomena. 